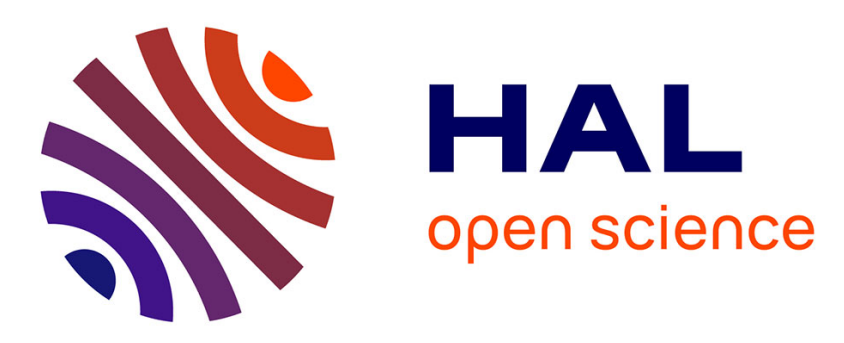

\title{
Error Modelling and Experimental Validation of a Planar 3-PPR Parallel Manipulator with Joint Clearances
}

\author{
Guanglei Wu, Bai Shaoping, Kepler Jørgen A., Stéphane Caro
}

\section{To cite this version:}

Guanglei Wu, Bai Shaoping, Kepler Jørgen A., Stéphane Caro. Error Modelling and Experimental Validation of a Planar 3-PPR Parallel Manipulator with Joint Clearances. Journal of Mechanisms and Robotics, 2012, 4 (4), pp.041011-1-8. 10.1115/1.4007487 . hal-00832640

\section{HAL Id: hal-00832640 \\ https://hal.science/hal-00832640}

Submitted on 11 Jun 2013

HAL is a multi-disciplinary open access archive for the deposit and dissemination of scientific research documents, whether they are published or not. The documents may come from teaching and research institutions in France or abroad, or from public or private research centers.
L'archive ouverte pluridisciplinaire HAL, est destinée au dépôt et à la diffusion de documents scientifiques de niveau recherche, publiés ou non, émanant des établissements d'enseignement et de recherche français ou étrangers, des laboratoires publics ou privés. 


\title{
Guanglei $\mathbf{W u}^{*}$ \\ e-mail: gwu@m-tech.aau.dk \\ Shaoping Bai \\ e-mail: shb@m-tech.aau.dk \\ Jørgen A. Kepler \\ e-mail: jk@m-tech.aau.dk \\ Error Modelling and Experimental Validation of a Planar 3-PPR Parallel Manipulator with Joint Clearances
}

Department of Mechanical and Manufacturing Engineering, Aalborg University, Fibigerstræde

16, DK-9220 Aalborg East, Denmark

\author{
Stéphane Caro \\ e-mail: stephane.caro@irccyn.ec-nantes.fr \\ Institut de Recherche en Communications et \\ Cybernétique de Nantes, France
}

\begin{abstract}
This paper deals with the error modelling and analysis of a 3-PPR planar parallel manipulator with joint clearances. The kinematics and the Cartesian workspace of the manipulator are analyzed. An error model is established with considerations of both configuration errors and joint clearances. Using this model, the upper bounds and distributions of the pose errors for this manipulator are established. The results are compared with experimental measurements and show the effectiveness of the error prediction model.
\end{abstract}

\section{Introduction}

The planar parallel manipulators (PPMs) with three identical kinematic chains are special parallel manipulators (PMs), whose motion is confined in a plane. For this type of PM, the error modelling and analysis are important for both design and control in order to utilize the PMs potential of high accuracy in applications.

A number of works on accuracy analysis of parallel mechanisms can be found in the literature. Ryu et al. derived a volumetric error model and a total error transformation matrix from a differential inverse kinematic equation, which includes all kinematic error sources [1]. Liu et al. reported an approach of geometric error modeling for lower mobility manipulators by explicitly separating the compensatable and uncompensatable error sources affecting the pose accuracy [2]. Yu et al. reported a simple geometric approach to computing the exact local maximum position and orientation error by illustrating several different types of 3-dof planar parallel robots [3]. $\mathrm{S}$. Briot et al. proposed a method based on geometric approach for detailed error analysis of a fully-parallel robot with three translations and one rotation that brings valuable understanding of the error amplification problem [4].

Research focusing on the influence of joint clearances has been reported too. Lin and Chen proposed a homogeneous error transformation matrix to assess the effects of joint clearances on pose errors [5]. Ting et al. presented a simple method to identify the worst position and direction errors due to the joint clearance of linkages and manipulators, which offers a geometrical model to warranty the precision of a mechanism [6]. Fogarasy and Smith utilized the derivatives of the closure equations to obtain a first order approximation of the output error, which is called the Jacobian method [7]. Regarding the errors of universal and spherical joints due to clearances as a part of link errors, Lim et al. [8] analyzed the dynamic error of a cubic parallel mechanism by using its forward kinematics. Castelli and Venanzi applied the virtual work principle to determine the position of the end-effector when a given external load is applied $[9,10]$. Meng et al. proposed an error model of PMs subject to joint clearances by formulating the error prediction model as a standard convex optimization problem [11], of which the constraints are formed through a set of inequalities about the joint clearances. A general error prediction model considering joint clearances was established for serial and parallel manipulators by

${ }^{*}$ Corresponding author. means of differential screw theory in [12]. It was used to analyze the kinematic sensitivity of a 3-PPR parallel manipulator to joint clearances in [13]. Wei and Simaan proposed an approach for designing inexpensive planar parallel robots with prescribed backlash-free workspace by using preloaded flexible joints to replace the passive joints [14]. Among the sources of errors, the influence of assembly and manufacturing errors and actuation errors can be eliminated as indicated in [5, 15-17] by calibration, except joint clearances due to its low repeatability. It means that the pose errors due to joint clearances require a special consideration. Simple and valid methods of error modelling for PPMs are needed for accuracy analysis.

In this paper, the error analysis of PPMs is studied with consideration of both configuration errors and joint clearances. An error model is established, upon which the maximal error problem was transformed into an optimization problem. The distributions of global maximal pose errors in the prescribed workspace can be formulated effectively. Moreover, the error model based on the joint clearances was validated experimentally. The work was conducted for a novel 3-PPR PPM with a non-symmetrical base [18], which has a larger workspace and the same level of motion accuracy compared to the traditional symmetrical PPMs.

This paper is organized as follows. The architecture of the manipulator under study is presented in Sec. 2. The kinematics and Cartesian workspace are analyzed in Sec. 3. The error prediction model is established in Sec. 4. Sections 5 and 6 present the experimental validation, in which measured results are compared with the simulations. The work is concluded in Sec. 7.

\section{Manipulator Under Study}

Figure 1 presents the CAD model of the planar 3-PPR parallel manipulator with a rigid equilateral triangle-shape moving platform (MP). Here and throughout this paper, $\mathrm{P}$ and $\mathrm{R}$ stand for prismatic and revolute joints, respectively. An underlined letter indicates an actuated joint. Each leg is driven by a CAL35 actuator, a high resolution linear motor built with an encoder of 5 microns accuracy from SMAC company [19]. A THK linear guide of model HRW17 is used as the active prismatic joint $\underline{P}$. A linear bearing mounted on the slider of the linear guide is used as the passive prismatic joint in each leg. The ball joints in Fig. 1 are preloaded, of which joint clearance does not exist. For the built physical prototype, the end-effector can also be replaced by a disk-shape MP with ordinary revolute joints to 


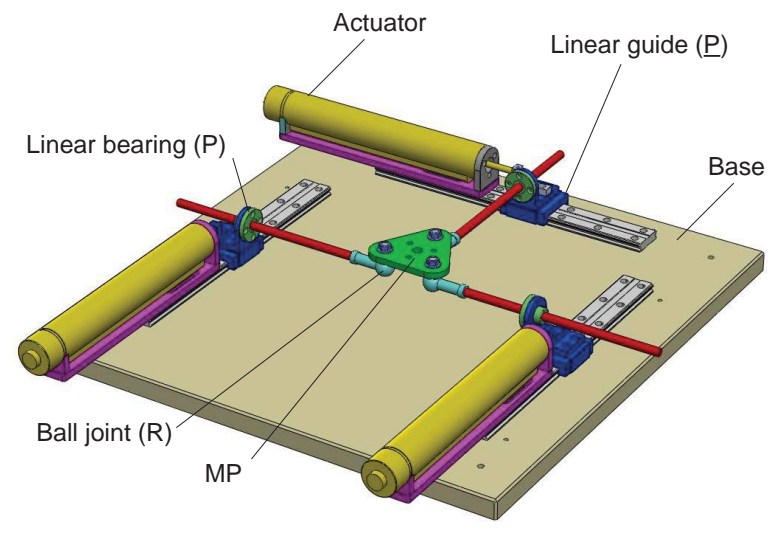

Fig. 1 CAD model of a 3-PPR PPM.

couple the three legs, but this introduces more error sources due to the clearances between the pin and the hole of the revolute joint.

The parameterization of the 3-PPR PPM is illustrated in Fig. 2 , where $A_{i}, i=1,2,3$, are fixed points on the base. The $x$-axis of the coordinate system $\mathcal{F}_{b}$ is parallel to the segment $A_{1} A_{2}$. The origin $P$ of the coordinate system $\mathcal{F}_{p}$ is located at the geometric center of the triangle $\triangle D_{1} D_{2} D_{3}$ on the moving platform and the $X$-axis is parallel to the segment $D_{1} D_{2}$, where $D_{i}, i=1,2,3$, are the centers of the revolute joints. The translational and orientational displacements of the MP are denoted by $\mathbf{p}$ and $\phi$, where $\mathbf{p}=[x, y], x$ and $y$ being the Cartesian coordinates of point $P$ in $\mathcal{F}_{b}$.

\section{Kinematic Modelling of the 3-PPR PPM}

The kinematic modelling of the manipulator is described in this section. The workspace and singularities of the manipulator are also analyzed based on its closure equations.

From the closed-loop kinematic chains $O-A_{i}-B_{i}-C_{i}-D_{i}-$ $P-O$ shown in Fig. 2, the position vector of point $P$ can be expressed in $\mathcal{F}_{b}$ as follows,

$$
\mathbf{p}=a_{i} \mathbf{h}_{i}+s_{i} \mathbf{u}_{i}+d_{i} \mathbf{v}_{i}+l_{i} \mathbf{w}_{i}+r_{i} \mathbf{k}_{i}, i=1,2,3
$$

with

$$
\begin{aligned}
& \mathbf{h}_{i}=\left[\begin{array}{c}
\cos \alpha_{i} \\
\sin \alpha_{i}
\end{array}\right], \mathbf{u}_{i}=\left[\begin{array}{c}
\cos \beta_{i}^{\prime} \\
\sin \beta_{i}^{\prime}
\end{array}\right], \mathbf{v}_{i}=\left[\begin{array}{c}
\cos \gamma_{i}^{\prime} \\
\sin \gamma_{i}^{\prime}
\end{array}\right], \\
& \mathbf{w}_{i}=\left[\begin{array}{c}
\cos \theta_{i}^{\prime} \\
\sin \theta_{i}^{\prime}
\end{array}\right], \mathbf{k}_{i}=\left[\begin{array}{c}
\cos \left(\phi+\psi_{i}\right) \\
\sin \left(\phi+\psi_{i}\right)
\end{array}\right]
\end{aligned}
$$

and

$$
\beta_{i}^{\prime}=\alpha_{i}+\beta_{i}, \gamma_{i}^{\prime}=\alpha_{i}+\beta_{i}+\gamma_{i}, \theta_{i}^{\prime}=\alpha_{i}+\beta_{i}+\gamma_{i}+\theta_{i}
$$

The inverse kinematics of the manipulator can be derived from Eqn. (1),

$$
\begin{aligned}
s_{i} & =\left(\mathbf{w}_{i}^{T} \mathbf{E} \mathbf{u}_{i}\right)^{-1} \mathbf{w}_{i}^{T} \mathbf{E}\left(\mathbf{p}-a_{i} \mathbf{h}_{i}-d_{i} \mathbf{v}_{i}-r_{i} \mathbf{k}_{i}\right) \\
l_{i} & =\left(\mathbf{u}_{i}^{T} \mathbf{E} \mathbf{w}_{i}\right)^{-1} \mathbf{u}_{i}^{T} \mathbf{E}\left(\mathbf{p}-a_{i} \mathbf{h}_{i}-d_{i} \mathbf{v}_{i}-r_{i} \mathbf{k}_{i}\right)
\end{aligned}
$$

matrix $\mathbf{E}$ is the right angle rotation matrix defined as

$$
\mathbf{E}=\left[\begin{array}{cc}
0 & -1 \\
1 & 0
\end{array}\right]
$$

Equation (1) establishes a system of six equations. The forward displacements can be solved by virtue of analytical method. The

\begin{tabular}{c|ccccc}
\hline \hline$i$ & $\alpha_{i}[\mathrm{rad}]$ & $\beta_{i}^{\prime}[\mathrm{rad}]$ & $\gamma_{i}^{\prime}, \theta_{i}^{\prime}[\mathrm{rad}]$ & $\psi_{i}[\mathrm{rad}]$ & $d_{i}[\mathrm{~mm}]$ \\
\hline 1 & -2.781 & $\pi / 2$ & 0 & $\pi / 6$ & 114 \\
\hline 2 & -0.360 & $\pi / 2$ & $\pi$ & $5 \pi / 6$ & 27 \\
\hline 3 & 1.751 & 0 & $-\pi / 2$ & $3 \pi / 2$ & 42 \\
\hline \hline
\end{tabular}

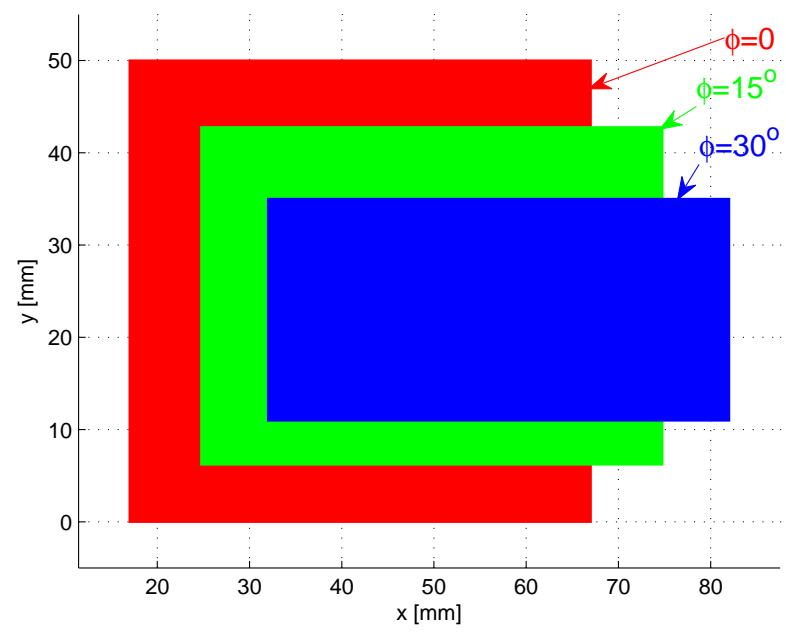

Fig. 3 Constant-orientation workspaces.

velocity expression of the manipulator can be derived from Eqn. (1) as below

$$
\mathbf{A}\left[\begin{array}{c}
\dot{\mathbf{p}} \\
\dot{\phi}
\end{array}\right]=\mathbf{B} \dot{\mathbf{s}}
$$

with

$$
\begin{aligned}
& \mathbf{A}=\left[\begin{array}{c}
\mathbf{w}_{1}^{T} \mathbf{E}^{T}-r_{1} \mathbf{w}_{1}^{T} \mathbf{k}_{1} \\
\mathbf{w}_{2}^{T} \mathbf{E}^{T}-r_{2} \mathbf{w}_{2}^{T} \mathbf{k}_{2} \\
\mathbf{w}_{3}^{T} \mathbf{E}^{T}-r_{3} \mathbf{w}_{3}^{T} \mathbf{k}_{3}
\end{array}\right] \\
& \mathbf{B}=\operatorname{diag}\left[\begin{array}{|l}
\mathbf{w}_{1}^{T} \mathbf{E}^{T} \mathbf{u}_{1} \mathbf{w}_{2}^{T} \mathbf{E}^{T} \mathbf{u}_{2} \mathbf{w}_{3}^{T} \mathbf{E}^{T} \mathbf{u}_{3}
\end{array}\right] \\
& \dot{\mathbf{s}}=\left[\begin{array}{lll}
\dot{s}_{1} & \dot{s}_{2} & \dot{s}_{3}
\end{array}\right]^{T}
\end{aligned}
$$

where $\mathbf{A}$ and $\mathbf{B}$ are the forward and backward Jacobians of the manipulator, respectively. The kinematic Jacobian matrix $\mathbf{J}$ of the manipulator takes the form:

$$
\mathbf{J}=\mathbf{A}^{-1} \mathbf{B}
$$

Matrix $\mathbf{A}$ is singular, i.e., the manipulator reaches a parallel singularity, when $\phi= \pm \pi / 2$. Matrix $\mathbf{B}$ is never singular, namely, the manipulator is free of serial singularity.

The reachable area of the moving platform with a constant orientation can be obtained geometrically by means of searching method $[13,18]$, where the inverse kinematics model, namely, Eqns. (2a) and (2b), establish a system of 12 inequations by virtue of the joint motion limits to formulate the motion constraints of the MP. With the parameters shown in Table 1 and $r_{i}=r=30 \mathrm{~mm}, a_{i}=$ $192.34 \mathrm{~mm}, i=1,2,3$, constant-orientation Cartesian workspaces for three orientations of the MP are illustrated in Fig. 3.

\section{Error Modelling of a 3-PPR PPM}

Here, a methodology introduced in [20] was used to derive the error model of the MP pose with regard to variations in the actuated 


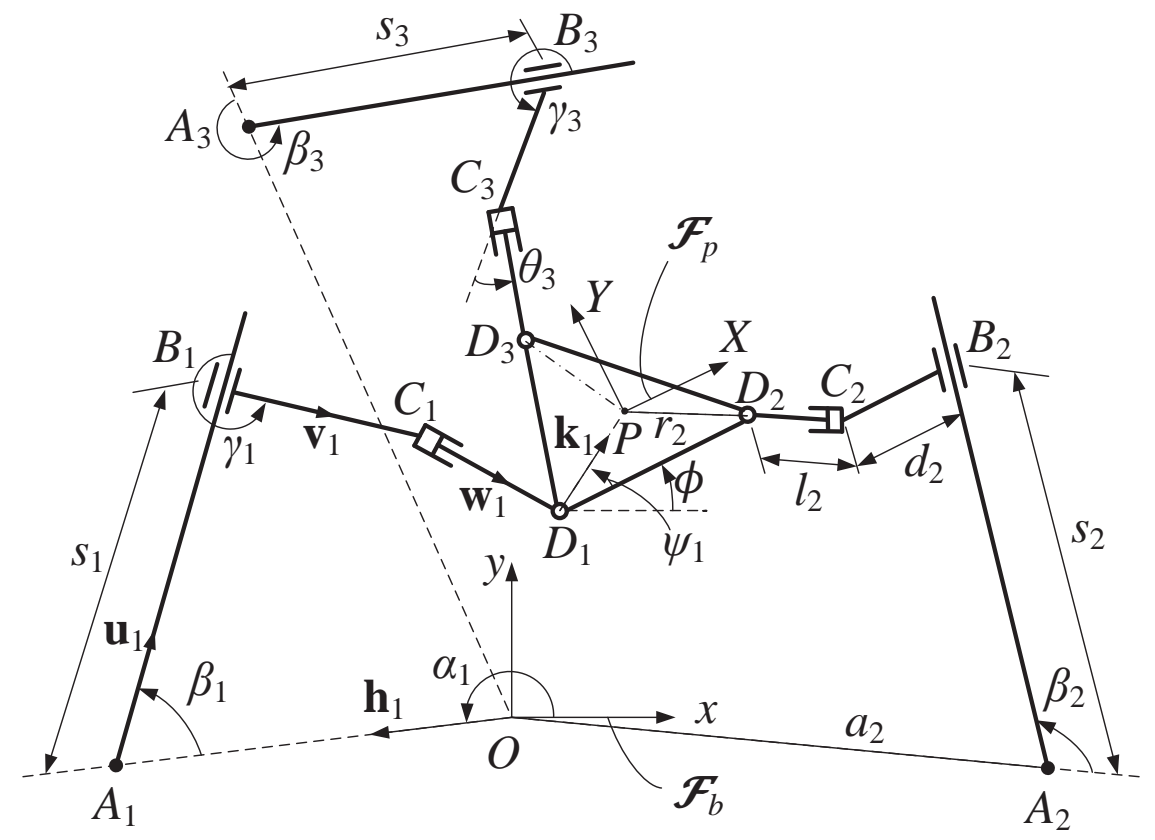

Fig. 2 Parameterization of the 3-PPR PPM.

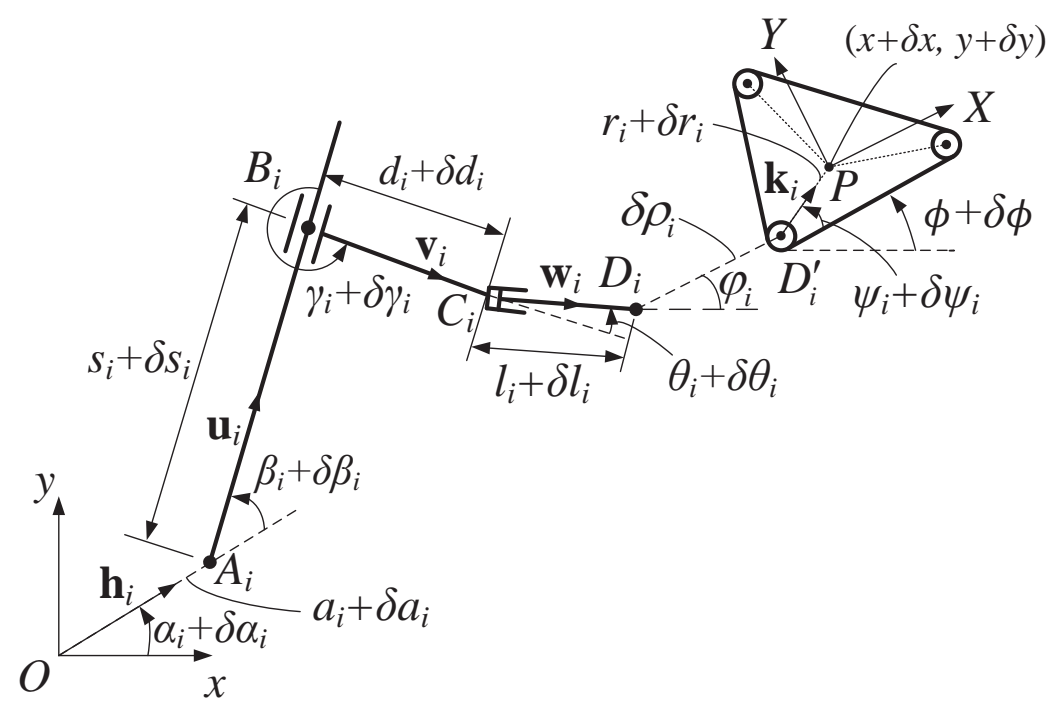

Fig. 4 Parameterization of the $i$ th leg.

and passive joints as well as in the Cartesian coordinates of points $A_{i}, B_{i}, C_{i}$ and $D_{i}, i=1,2,3$.

4.1 Error Prediction Model. The clearance in the revolute joint between the $i$ th leg and the moving platform is characterized by the small displacement between points $D_{i}$ and point $D_{i}^{\prime}$ as shown in Fig. 4. Upon differentiation of Eqn. (1), we obtain the positioning error of point $P$ with respect to each leg

$$
\begin{aligned}
\delta \mathbf{p}= & \delta a_{i} \mathbf{h}_{i}+a_{i} \delta \alpha_{i} \mathbf{E} \mathbf{h}_{i}+\delta s_{i} \mathbf{u}_{i}+s_{i} \delta \beta_{i}^{\prime} \mathbf{E} \mathbf{u}_{i}+\delta d_{i} \mathbf{v}_{i} \\
& +d_{i} \delta \gamma_{i}^{\prime} \mathbf{E} \mathbf{v}_{i}+\delta l_{i} \mathbf{w}_{i}+l_{i} \delta \theta_{i}^{\prime} \mathbf{E} \mathbf{w}_{i}+\delta \rho_{i} \mathbf{n}_{i} \\
& +\delta r_{i} \mathbf{k}_{i}+r_{i}\left(\delta \phi+\delta \psi_{i}\right) \mathbf{E} \mathbf{k}_{i}, i=1,2,3
\end{aligned}
$$

where

$$
\begin{aligned}
& \delta \beta_{i}^{\prime}=\delta \alpha_{i}+\delta \beta_{i} \\
& \delta \gamma_{i}^{\prime}=\delta \alpha_{i}+\delta \beta_{i}+\delta \gamma_{i} \\
& \delta \theta_{i}^{\prime}=\delta \alpha_{i}+\delta \beta_{i}+\delta \gamma_{i}+\delta \theta_{i}
\end{aligned}
$$

where $\delta \mathbf{p}$ and $\delta \phi$ are the positioning and orientation errors of the moving platform expressed in $\mathcal{F}_{b}$, respectively. Moreover, $\delta a_{i}, \delta \alpha_{i}$, $\delta s_{i}, \delta \beta_{i}, \delta d_{i}, \delta \gamma_{i}, \delta l_{i}, \delta \theta_{i}, \delta r_{i}$ and $\delta \psi_{i}$ denote variations in the geometric parameters illustrated in Fig. 4. In addition, $\delta \rho_{i}$ is a small displacement between point $D_{i}$ and point $D_{i}^{\prime}$ due to the clearance in the $i$ th revolute joint and $\mathbf{n}_{i}=\left[\cos \varphi_{i}, \sin \varphi_{i}\right]^{T}$, as illustrated in Fig. 5(b). Substituting Eqn. (8) into (6) and eliminating the idle 
variation $\delta l_{i}$ lead to

$$
\begin{aligned}
& \mathbf{w}_{i}^{T} \mathbf{E}^{T} \delta \mathbf{p}=\delta a_{i} \mathbf{w}_{i}^{T} \mathbf{E}^{T} \mathbf{h}_{i}+\delta \alpha_{i}\left[\mathbf{w}_{i}^{T}\left(a_{i} \mathbf{h}_{i}+s_{i} \mathbf{u}_{i}+d_{i} \mathbf{v}_{i}\right)+l_{i}\right] \\
& \quad+\delta s_{i} \mathbf{w}_{i}^{T} \mathbf{E}^{T} \mathbf{u}_{i}+\delta \beta_{i}\left[\mathbf{w}_{i}^{T}\left(s_{i} \mathbf{u}_{i}+d_{i} \mathbf{v}_{i}\right)+l_{i}\right] \\
& \quad+\delta d_{i} \mathbf{w}_{i}^{T} \mathbf{E}^{T} \mathbf{v}_{i}+\delta \gamma_{i}\left(d_{i} \mathbf{w}_{i}^{T} \mathbf{v}_{i}+l_{i}\right)+l_{i} \delta \theta_{i} \\
& \quad+\delta \rho_{i} \mathbf{w}_{i}^{T} \mathbf{E}^{T} \mathbf{n}_{i}+\delta r_{i} \mathbf{w}_{i}^{T} \mathbf{E}^{T} \mathbf{k}_{i}+r_{i}\left(\delta \phi+\delta \psi_{i}\right) \mathbf{w}_{i}^{T} \mathbf{k}_{i}
\end{aligned}
$$

Equation (8) can be cast in vector form:

$$
\begin{aligned}
\mathbf{A}\left[\begin{array}{c}
\delta x \\
\delta y \\
\delta \phi
\end{array}\right]= & \mathbf{H}_{a}\left[\begin{array}{l}
\delta a_{1} \\
\delta a_{2} \\
\delta a_{3}
\end{array}\right]+\mathbf{H}_{\alpha}\left[\begin{array}{l}
\delta \alpha_{1} \\
\delta \alpha_{2} \\
\delta \alpha_{3}
\end{array}\right]+\mathbf{B}\left[\begin{array}{l}
\delta s_{1} \\
\delta s_{2} \\
\delta s_{3}
\end{array}\right] \\
& +\mathbf{H}_{\beta}\left[\begin{array}{l}
\delta \beta_{1} \\
\delta \beta_{2} \\
\delta \beta_{3}
\end{array}\right]+\mathbf{H}_{d}\left[\begin{array}{l}
\delta d_{1} \\
\delta d_{2} \\
\delta d_{3}
\end{array}\right]+\mathbf{H}_{\gamma}\left[\begin{array}{l}
\delta \gamma_{1} \\
\delta \gamma_{2} \\
\delta \gamma_{3}
\end{array}\right] \\
& +\mathbf{H}_{\theta}\left[\begin{array}{l}
\delta \theta_{1} \\
\delta \theta_{2} \\
\delta \theta_{3}
\end{array}\right]+\mathbf{H}_{\rho}\left[\begin{array}{l}
\delta \rho_{1} \\
\delta \rho_{2} \\
\delta \rho_{3}
\end{array}\right] \\
& +\mathbf{H}_{r}\left[\begin{array}{l}
\delta r_{1} \\
\delta r_{2} \\
\delta r_{3}
\end{array}\right]+\mathbf{H}_{\psi}\left[\begin{array}{l}
\delta \psi_{1} \\
\delta \psi_{2} \\
\delta \psi_{3}
\end{array}\right]
\end{aligned}
$$

where all $\mathbf{H}_{q}, q \in\{a, \alpha, \beta, d, \gamma, \theta, \rho, r, \psi\}$, are $3 \times 3$ matrices as given in Appendix A. Moreover, assuming that $\mathbf{A}$ is nonsingular, the multiplication of Eqn. (9) by $\mathbf{A}^{-1}$ leads to

$$
\begin{aligned}
& {\left[\begin{array}{c}
\delta x \\
\delta y \\
\delta \phi
\end{array}\right]=} \mathbf{J}_{a}\left[\begin{array}{l}
\delta a_{1} \\
\delta a_{2} \\
\delta a_{3}
\end{array}\right]+\mathbf{J}_{\alpha}\left[\begin{array}{l}
\delta \alpha_{1} \\
\delta \alpha_{2} \\
\delta \alpha_{3}
\end{array}\right]+\mathbf{J}\left[\begin{array}{l}
\delta s_{1} \\
\delta s_{2} \\
\delta s_{3}
\end{array}\right]+\mathbf{J}_{\beta}\left[\begin{array}{l}
\delta \beta_{1} \\
\delta \beta_{2} \\
\delta \beta_{3}
\end{array}\right] \\
&+\mathbf{J}_{d}\left[\begin{array}{l}
\delta d_{1} \\
\delta d_{2} \\
\delta d_{3}
\end{array}\right]+\mathbf{J}_{\gamma}\left[\begin{array}{l}
\delta \gamma_{1} \\
\delta \gamma_{2} \\
\delta \gamma_{3}
\end{array}\right]+\mathbf{J}_{\theta}\left[\begin{array}{l}
\delta \theta_{1} \\
\delta \theta_{2} \\
\delta \theta_{3}
\end{array}\right] \\
&+\mathbf{J}_{\rho}\left[\begin{array}{l}
\delta \rho_{1} \\
\delta \rho_{2} \\
\delta \rho_{3}
\end{array}\right]+\mathbf{J}_{r}\left[\begin{array}{l}
\delta r_{1} \\
\delta r_{2} \\
\delta r_{3}
\end{array}\right]+\mathbf{J}_{\psi}\left[\begin{array}{l}
\delta \psi_{1} \\
\delta \psi_{2} \\
\delta \psi_{3}
\end{array}\right]
\end{aligned}
$$

with

$$
\mathbf{J}_{q}=\mathbf{A}^{-\mathbf{1}} \mathbf{H}_{q}, q \in\{a, \alpha, \beta, d, \gamma, \theta, \rho, r, \psi\}
$$

where $\mathbf{J}$ and $\mathbf{J}_{q}$ are the sensitivity coefficients of the MP pose of the manipulator to variations in terms of coordinates of each link [20]. It will be more useful to find the sensitivity coefficients in the coordinates of all joint positions, namely, points $A_{i}, B_{i}, C_{i}$ and $D_{i}$. By making use of

$$
\begin{aligned}
& {\left[\begin{array}{l}
\delta a_{i x} \\
\delta a_{i y}
\end{array}\right]=\left[\begin{array}{cc}
\cos \alpha_{i} & -a_{i} \sin \alpha_{i} \\
\sin \alpha_{i} & a_{i} \cos \alpha_{i}
\end{array}\right]\left[\begin{array}{c}
\delta a_{i} \\
\delta \alpha_{i}
\end{array}\right]} \\
& {\left[\begin{array}{l}
\delta b_{i x} \\
\delta b_{i y}
\end{array}\right]=\left[\begin{array}{cc}
\cos \beta_{i} & -s_{i} \sin \beta_{i} \\
\sin \beta_{i} & s_{i} \cos \beta_{i}
\end{array}\right]\left[\begin{array}{c}
0 \\
\delta \beta_{i}
\end{array}\right]} \\
& {\left[\begin{array}{l}
\delta c_{i x} \\
\delta c_{i y}
\end{array}\right]=\left[\begin{array}{cc}
\cos \gamma_{i} & -d_{i} \sin \gamma_{i} \\
\sin \gamma_{i} & d_{i} \cos \gamma_{i}
\end{array}\right]\left[\begin{array}{c}
\delta d_{i} \\
\delta \gamma_{i}
\end{array}\right]} \\
& {\left[\begin{array}{l}
\delta d_{i x} \\
\delta d_{i y}
\end{array}\right]=\left[\begin{array}{cc}
\cos \psi_{i} & -r_{i} \sin \psi_{i} \\
\sin \psi_{i} & r_{i} \cos \psi_{i}
\end{array}\right]\left[\begin{array}{c}
\delta r_{i} \\
\delta \psi_{i}
\end{array}\right]}
\end{aligned}
$$

Eqn. (10) is transformed as

$$
\begin{gathered}
{\left[\begin{array}{l}
\delta \mathbf{p} \\
\delta \phi
\end{array}\right]=\mathbf{J}\left[\begin{array}{l}
\delta s_{1} \\
\delta s_{2} \\
\delta s_{3}
\end{array}\right]+\mathbf{J}_{\theta}\left[\begin{array}{l}
\delta \theta_{1} \\
\delta \theta_{2} \\
\delta \theta_{3}
\end{array}\right]+\mathbf{J}_{\rho}\left[\begin{array}{l}
\delta \rho_{1} \\
\delta \rho_{2} \\
\delta \rho_{3}
\end{array}\right]+\mathbf{J}_{A}\left[\begin{array}{l}
\delta a_{1 x} \\
\delta a_{1 y} \\
\delta a_{2 x} \\
\delta a_{2 y} \\
\delta a_{3 x} \\
\delta a_{3 y}
\end{array}\right]} \\
+\mathbf{J}_{B}\left[\begin{array}{l}
\delta b_{1 x} \\
\delta b_{1 y} \\
\delta b_{2 x} \\
\delta b_{2 y} \\
\delta b_{3 x} \\
\delta b_{3 y}
\end{array}\right]+\mathbf{J}_{C}\left[\begin{array}{l}
\delta c_{1 x} \\
\delta c_{1 y} \\
\delta c_{2 x} \\
\delta c_{2 y} \\
\delta c_{3 x} \\
\delta c_{3 y}
\end{array}\right]+\mathbf{J}_{D}\left[\begin{array}{l}
\delta d_{1 x} \\
\delta d_{1 y} \\
\delta d_{2 x} \\
\delta d_{2 y} \\
\delta d_{3 x} \\
\delta d_{3 y}
\end{array}\right]
\end{gathered}
$$

where $\delta a_{i x}$ and $\delta a_{i y}\left(\delta b_{i x}\right.$ and $\delta b_{i y}, \delta c_{i x}$ and $\delta c_{i y}$, resp.) are the positioning errors of point $A_{i}\left(B_{i}, C_{i}\right.$, resp.), $i=1,2,3$, along $x$ - and $y$-axis, namely, the variations in the Cartesian coordinates. Notice that $\delta d_{i x}$ and $\delta d_{i y}$ denote the positioning errors of points $D_{i}^{\prime}$ along $X$ and $Y$-axis, namely, the variations in the Cartesian coordinates of $D_{i}^{\prime}$. The $3 \times 6$ matrices $\mathbf{J}_{A}, \mathbf{J}_{B}, \mathbf{J}_{C}$ and $\mathbf{J}_{D}$ can be found in Appendix $A$. Equation (13) can be written in the following form,

$$
\left[\begin{array}{c}
\delta \mathbf{p} \\
\delta \phi
\end{array}\right]=\mathbf{J}_{e r r} \boldsymbol{\delta}_{v a r}
$$

where

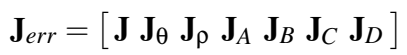

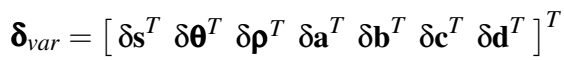

with

$$
\begin{gathered}
\delta \mathbf{s}=\left[\begin{array}{l}
\delta s_{1} \\
\delta s_{2} \\
\delta s_{3}
\end{array}\right], \delta \boldsymbol{\theta}=\left[\begin{array}{l}
\delta \theta_{1} \\
\delta \theta_{2} \\
\delta \theta_{3}
\end{array}\right], \delta \boldsymbol{\rho}=\left[\begin{array}{l}
\delta \rho_{1} \\
\delta \rho_{2} \\
\delta \rho_{3}
\end{array}\right], \delta \mathbf{e}=\left[\begin{array}{l}
\delta \mathbf{e}_{1} \\
\delta \mathbf{e}_{2} \\
\delta \mathbf{e}_{3}
\end{array}\right] \\
\delta \mathbf{e}_{i}=\left[\delta e_{i x} \delta e_{i y}\right]^{T}, e \in\{a, b, c, d\}, i=1,2,3
\end{gathered}
$$

where $\mathbf{J}_{\text {err }}$ is the global sensitivity Jacobian matrix and $\boldsymbol{\delta}_{\text {var }}$ is a vector containing all variations. For a given posture, all submatrices except $\mathbf{J}_{\rho}$ are known.

4.2 Modelling the Joint Clearances. Figure 5 illustrates the assembly errors and clearances in the prismatic and revolute joints. $\delta d_{a i}, \delta \beta_{i}, \delta \gamma_{a i}, \delta \theta_{a i}, i=1,2,3$, are the assembly errors and $\delta d_{i x}, \delta d_{i y}$ correspond to the manufacturing errors. Moreover, $\Delta \sigma_{g i}$, $\Delta \tau_{g i}, \Delta \tau_{b i}, \Delta \rho_{i}$ are the displacements due to joint clearances. Then we have:

$$
\delta d_{i}=\delta d_{a i}+\Delta \sigma_{g i}, \delta \gamma_{i}=\delta \gamma_{a i}+\Delta \tau_{g i}, \delta \theta_{i}=\delta \theta_{a i}+\Delta \tau_{b i}
$$

The errors due to the clearances in the linear guides are characterized by the following constraints [11]:

$$
\begin{aligned}
& -2 \varepsilon_{g i} \leq L_{g} \Delta \tau_{g i}+2 \Delta \sigma_{g i} \leq 2 \varepsilon_{g i} \\
& -2 \varepsilon_{g i} \leq-L_{g} \Delta \tau_{g i}+2 \Delta \sigma_{g i} \leq 2 \varepsilon_{g i}
\end{aligned}
$$

where $\varepsilon_{g i}$ specifies the lateral clearance and $L_{g}$ is the length of the linear guide block. Alternatively, the errors in the linear bearing are constrained by the following condition:

$$
-\varepsilon_{\theta i} \leq \Delta \tau_{b i} \leq \varepsilon_{\theta i}
$$



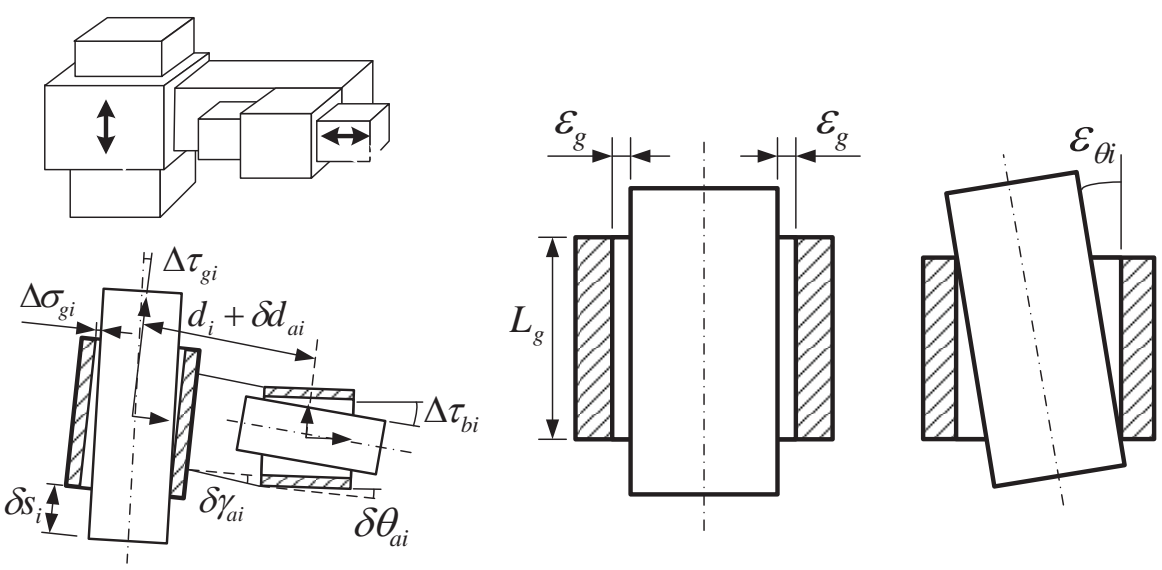

(a)
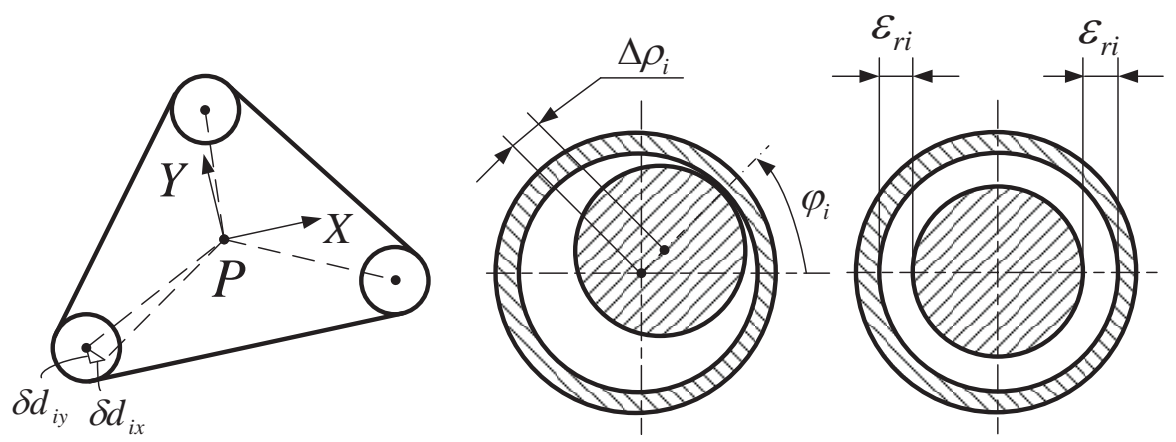

(b)

Fig. 5 Geometric errors and joint clearances related to the $i$ th leg: (a) prismatic joint, (b) revolute joint.

where $\varepsilon_{\theta i}$ is the upper bound of $\Delta \tau_{b i}$. Figure 5(a) demonstrates the tolerances of the linear guides and bearing. The clearance in the three revolute joints meet the following constraint:

$$
0 \leq \Delta \rho_{i} \leq \varepsilon_{r i}, i=1,2,3
$$

where $\varepsilon_{r i}$ is the range of variations $\Delta \rho_{i}$ due to the joint clearance shown in Fig. 5(b).

4.3 Maximum Pose Errors of the Moving Platform with Joint Clearances. The pose errors due to the assembly and actuation errors can be determined from Eqn. (14), while the errors due to joint clearances will be solved by virtue of an optimization method. When only joint clearances are considered, the relationship between the pose errors and joint clearances becomes:

$$
\left[\begin{array}{l}
\delta \mathbf{p} \\
\delta \phi
\end{array}\right]=\mathbf{J}_{e r r}^{c} \boldsymbol{\delta}_{v a r}^{c}
$$

with

$$
\begin{aligned}
& \mathbf{J}_{\text {err }}^{c}=\left[\begin{array}{llll}
\mathbf{J}_{\theta} & \mathbf{J}_{d} & \mathbf{J}_{\gamma} & \mathbf{J}_{\rho}
\end{array}\right]_{3 \times 12}=\left[\begin{array}{llll}
\mathbf{J}_{\text {err }, x}^{c} & \mathbf{J}_{\text {err }, y}^{c} & T & \mathbf{J}_{\text {err }, \phi}^{c}
\end{array}\right]^{T} \\
& \boldsymbol{\delta}_{\text {var }}^{c}=\left[\Delta \tau_{b 1} \Delta \tau_{b 2} \Delta \tau_{b 3} \Delta \sigma_{g 1} \Delta \sigma_{g 2} \Delta \sigma_{g 3} \Delta \tau_{g 1} \Delta \tau_{g 2}\right. \\
& \left.\Delta \tau_{g 3} \Delta \rho_{1} \Delta \rho_{2} \Delta \rho_{3}\right]^{T}
\end{aligned}
$$

where $\mathbf{J}_{e r r, x}^{c}, \mathbf{J}_{\text {err }, y}^{c}, \mathbf{J}_{\text {err }, \phi}^{c}$ are three $1 \times 12$ submatrices corresponding to the first, the second and the third rows of $\mathbf{J}_{\text {err }}^{c}$. The maximum positioning error along $x$-axis, $y$-axis and the maximum orientation error of the MP, namely, $\delta x_{\max }, \delta y_{\max }$ and $\delta \phi_{\max }$, can be obtained by solving the following optimization problem:

$$
\begin{aligned}
\delta \zeta_{\max }^{2} \equiv & \max \left(\mathbf{J}_{\text {err }, \zeta}^{c} \boldsymbol{\delta}_{\text {var }}^{c}\right)^{2} \text { for } x, y, \phi \in \Omega \\
\text { S.T. } & -2 \varepsilon_{g i} \leq L_{g} \Delta \tau_{g i}+2 \Delta \sigma_{g i} \leq 2 \varepsilon_{g i} \\
& -2 \varepsilon_{g i} \leq-L_{g} \Delta \tau_{g i}+2 \Delta \sigma_{g i} \leq 2 \varepsilon_{g i} \\
& -\varepsilon_{\theta i} \leq \Delta \tau_{b i} \leq \varepsilon_{\theta i} \\
& 0 \leq \Delta \rho_{i} \leq \varepsilon_{r i} \\
& 0 \leq \varphi_{i} \leq 2 \pi \\
& i=1,2,3, \zeta \in\{x, y, \phi\}
\end{aligned}
$$

where $\Omega$ denotes the Cartesian workspace of the manipulator defined in Sec. 3. Optimization problem of Eqn. (21) aims at finding separately the maximum positioning errors along the $x$-axis and $y$-axis and the maximum orientation error of the moving platform. Note that the three maximum errors are subject to the same constraints, hence, the optimization problems are written in a generalized form.

The maximum positioning error $\delta p_{\max }$ is obtained by solving 
the following optimization problem:

$$
\begin{aligned}
\delta p_{\max }^{2} \equiv & \max \left(\mathbf{J}_{\text {err,p}}^{c} \boldsymbol{\delta}_{\text {var }}^{c}\right)^{T}\left(\mathbf{J}_{\text {err }, p}^{c} \boldsymbol{\delta}_{\text {var }}^{c}\right) \text { for } x, y, \phi \in \Omega \\
\text { S.T. } & -2 \varepsilon_{g i} \leq L_{g} \Delta \tau_{g i}+2 \Delta \sigma_{g i} \leq 2 \varepsilon_{g i} \\
& -2 \varepsilon_{g i} \leq-L_{g} \Delta \tau_{g i}+2 \Delta \sigma_{g i} \leq 2 \varepsilon_{g i} \\
& -\varepsilon_{\theta i} \leq \Delta \tau_{b i} \leq \varepsilon_{\theta i} \\
& 0 \leq \Delta \rho_{i} \leq \varepsilon_{r i} \\
& 0 \leq \varphi_{i} \leq 2 \pi \\
& i=1,2,3
\end{aligned}
$$

where $\mathbf{J}_{e r r, p}^{c}=\left[\begin{array}{llll}\mathbf{J}_{e r r, x}^{c} & T & \mathbf{J}_{\text {err }, y}^{c} & T\end{array}\right]^{T}$. The foregoing optimization problems are solved using the Matlab fmincon function. According to the product catalogues, the clearance in the lateral direction of the linear guide is equal to $3 \mu \mathrm{m}$, namely, $2 \varepsilon_{g i}=2 \varepsilon_{g}=3 \mu \mathrm{m}$. As a consequence, the errors due to the linear guides are negligible. $\delta a_{i x}$ and $\delta a_{i y}$ are set to zero too. Finally, the maximum position error and the maximum orientation error of the MP can be evaluated from Eqn. (21) and Eqn. (22) for any configuration of the manipulator by known joint clearances and geometric tolerances.

\section{Experimental Setup and Measurement Errors}

A main purpose of the work is to experimentally validate the error model developed. To this end, experiments have been conducted in which the position and orientation of the MP were measured with a vision-based system composed of a single CCD camera. The experimental setup is shown in Fig. 6(a) and its specifications are given hereafter:

- DVT 554c smart camera with $1280 \times 1024$ pixel resolution (7.4 $\mu \mathrm{m} \times 7.4 \mu \mathrm{m}$ pixels) from Cognex [21] was fixed right above the MP for pose measurements.

- Intellect 1.5.1, a vision software from Cognex [22], was used to establish the communication with the camera via data cable as shown in Fig. 6(b). The Blobs are used to locate markers on the MP.

With this system, the position and orientation measurement accuracies are equal to $0.01 \mathrm{~mm}$ and $0.01 \mathrm{deg}$, respectively.

5.1 Measurements. Before the measurements, the system was calibrated. A standard calibration paper with markers of $2 \mathrm{~cm}$ spacing from Cognex was used to establish the reference frame. The calibration method is described in the Intellect 1.5 Guide [22].

5.1.1 Assembly Errors. In measuring configuration error, the first linear guide was used as the $y$-axis of the reference frame, which means $\delta \beta_{1}=0$. The measurement is illustrated in Fig. 7. Four holes on each linear guide were used as the markers. $\delta \beta_{2}$ and $\delta \beta_{3}$ can be obtained by means of the Intellect software. Similarly, a perfect regular component with four uniformly distributed holes was used to measure the assembly errors $\delta \gamma_{a i}$ and $\delta \theta_{a i}$ by means of face to face alignments. The measured assembly errors are listed in Table 2

5.1.2 Joint Clearances. Figure 8 illustrates the method used to measure the clearance in the linear bearing. Pushing the right end of the shaft back and forth and measuring the difference of the

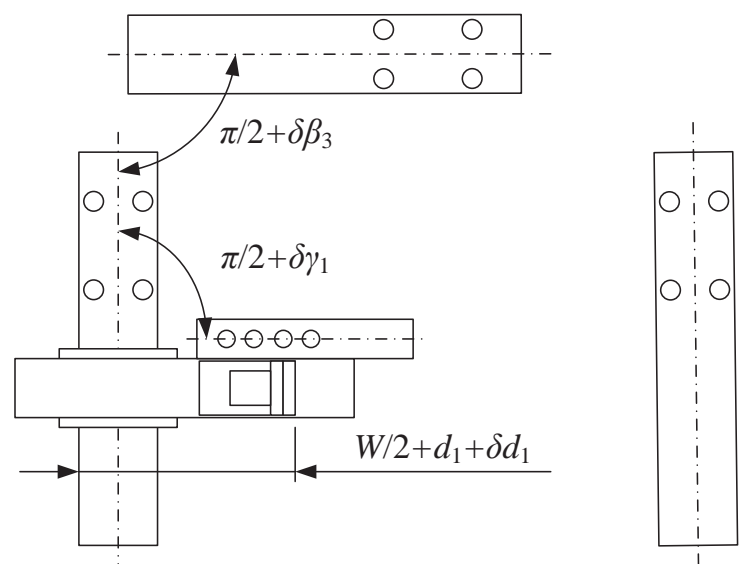

Fig. 7 Measurement of the assembly errors.

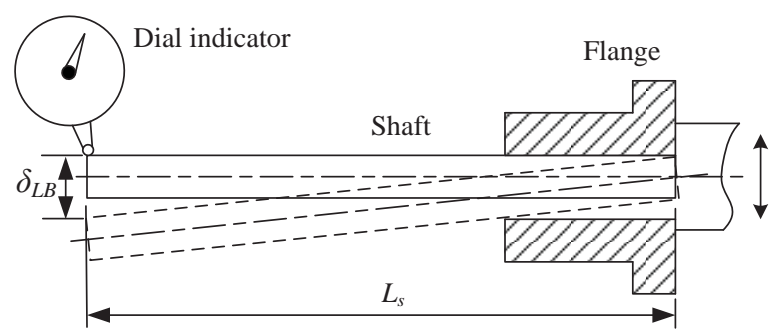

Fig. 8 Measurement of the angular clearance in the linear bearing.

Table 2 Measurements of Assembly Errors

\begin{tabular}{c|cccc}
\hline \hline$i$ & $\delta \beta_{i}[\mathrm{rad}]$ & $\delta \gamma_{a i}[\mathrm{rad}]$ & $\delta \theta_{a i}[\mathrm{rad}]$ & $\delta d_{a i}[\mathrm{~mm}]$ \\
\hline 1 & 0 & 0.016 & -0.028 & -0.12 \\
\hline 2 & 0.014 & 0.020 & -0.022 & -0.08 \\
\hline 3 & -0.010 & 0.020 & 0.034 & 0.15 \\
\hline \hline
\end{tabular}

two counts $\delta_{L B}$, the value of $\delta_{L B} / L_{S}$ was adopted as the bound of angular clearance. For the revolute joint clearances, the diameters of the joint pin and the cylinder were measured, respectively. The half value of the difference of the two measurements was adopted as the clearance bound. The bounds of the joint clearances were found as:

$$
\begin{aligned}
& \varepsilon_{\theta i}=\varepsilon_{\theta}=0.0012 \mathrm{rad}, i=1,2,3 \\
& \varepsilon_{r 1}=0.039 \mathrm{~mm}, \varepsilon_{r 2}=0.036 \mathrm{~mm}, \varepsilon_{r 3}=0.037 \mathrm{~mm}
\end{aligned}
$$

5.2 Pose Errors of MP. The measurements were conducted with two cases:

Case 1: a case with only clearances in the passive prismatic joints

Case 2: a case with clearances in both passive prismatic and revolute joints

The two cases were physically implemented with two different shapes for the moving platform in Sec. 2, respectively, namely, the equilateral triangle MP $(\triangle$-shape MP) and the disk-shape MP $(\bigcirc$ shape MP), which are associated with Cases 1 and 2 . We first fixed on the MP a calibration paper with $2 \times 2$ marks of $2 \mathrm{~cm}$ separation in Case 1 . Then, $i \times j$ uniformly distributed points were measured 


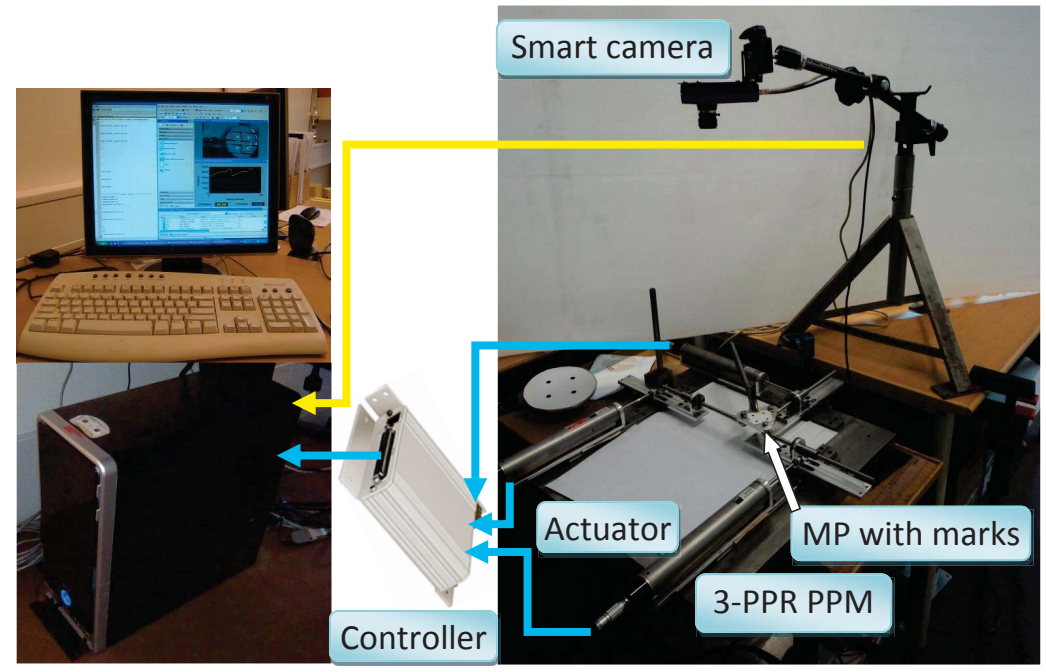

(a)

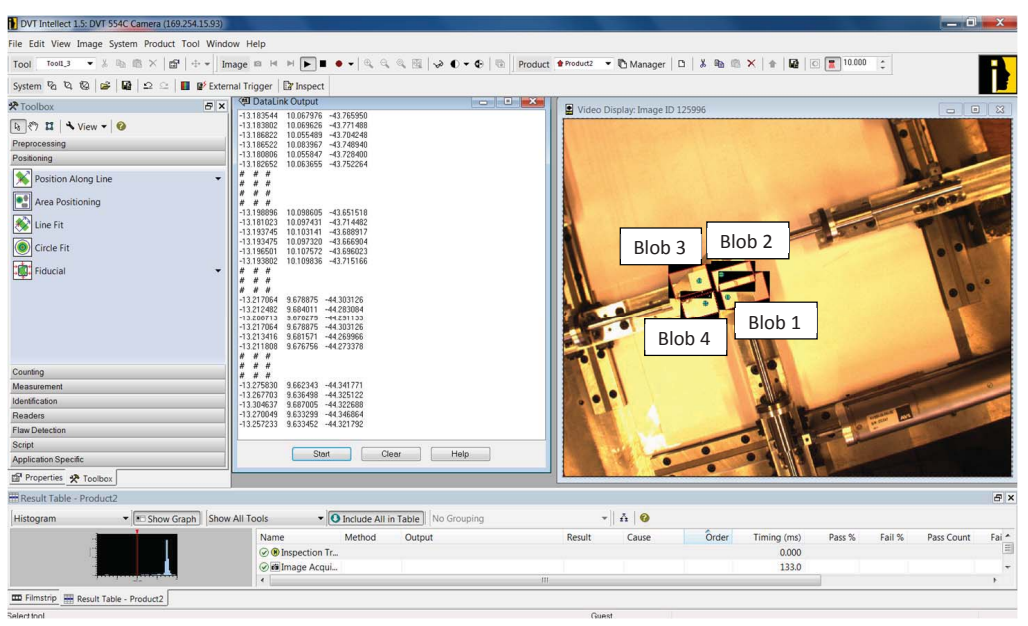

(b)

Fig. 6 A vision-based system for the moving platform pose measurement: (a) experimental setup, (b) measurement interface.

throughout the Cartesian workspace of the manipulator. During the measurements, the actuators were locked to eliminate the the errors in the actuators. At the $(i, j)$ point, the MP was slightly pushed bidirectionally along the $x$-axis, $y$-axis and rotated about the $z$-axis, respectively. The corresponding readings were noted as $(x, y, \phi)_{+t}^{i j}$ and $(x, y, \phi)_{-t}^{i j}, t \in\{x, y, r\}$, respectively. The measured positioning and orientation errors at the $(i, j)$ point are defined as:

$$
\begin{aligned}
& \delta x^{i j}=\max \left\{x_{+t}^{i j}, x_{-t}^{i j}\right\}-\min \left\{x_{+t}^{i j}, x_{-t}^{i j}\right\} \\
& \delta y^{i j}=\max \left\{y_{+t}^{i j}, y_{-t}^{i j}\right\}-\min \left\{y_{+t}^{i j}, y_{-t}^{i j}\right\} \\
& \delta \phi^{i j}=\max \left\{\phi_{+t}^{i j}, \phi_{-t}^{i j}\right\}-\min \left\{\phi_{+t}^{i j}, \phi_{-t}^{i j}\right\} \\
& \delta p^{i j}=\max \left\{\delta p_{t}^{i j}\right\}, \delta p_{t}^{i j}=\sqrt{\left(x_{+t}^{i j}-x_{-t}^{i j}\right)^{2}+\left(y_{+t}^{i j}-y_{-t}^{i j}\right)^{2}}
\end{aligned}
$$

\section{Results and Discussion}

In this section, the predicted maximum pose errors from the model and measured errors from the experiments are presented and compared.
6.1 Error Distributions for Case 1. Figure 9 represents the error distribution of the moving platform for a given orientation $\phi=0$. Figure 9(a) shows that the simulated $\delta x_{\max }$ is constant for a given $y$ coordinate and decreases slightly with the $y$-coordinate. $\delta x_{\max }$ is bounded between $0.196 \mathrm{~mm}$ and $0.256 \mathrm{~mm}$, while $\delta y_{\max }$ and $\delta \phi_{\max }$ are both constant, their values being equal to $0.100 \mathrm{~mm}$ and $0.221 \mathrm{deg}$, respectively. The $y$-coordinate of point $P$, the geometric center of the MP, and the orientation of the MP depend only on the first and second prismatic actuators because of the partial motion decoupling of the manipulator. Therefore, the maximum position error of the MP along the $y$-axis and its maximum rotation error occur when $\Delta \tau_{b i}, i=1,2$, reach their lower or upper bounds. As a result, both $\delta y_{\max }$ and $\delta \phi_{\max }$ remain constant throughout the Cartesian workspace of the manipulator. From Fig. 9(c), it is apparent that the maximum positioning error $\delta p_{\max }$ of the MP is symmetrical with respect to the $x$-axis. The root-mean-square deviation (RMSD) values between the simulations and measurements are equal to $50 \mu \mathrm{m}$, $30 \mu \mathrm{m}, 51 \mu \mathrm{m}$ and $0.057 \mathrm{deg}$, for $\delta x, \delta y, \delta p$ and $\delta \phi$, respectively. From Fig. 9, it is noteworthy that there is a good correlation between the measured positioning errors and the simulated ones. On the other hand, the differences between the measured orientation errors of the MP and the simulated ones are noticeable. To some extent, this is 


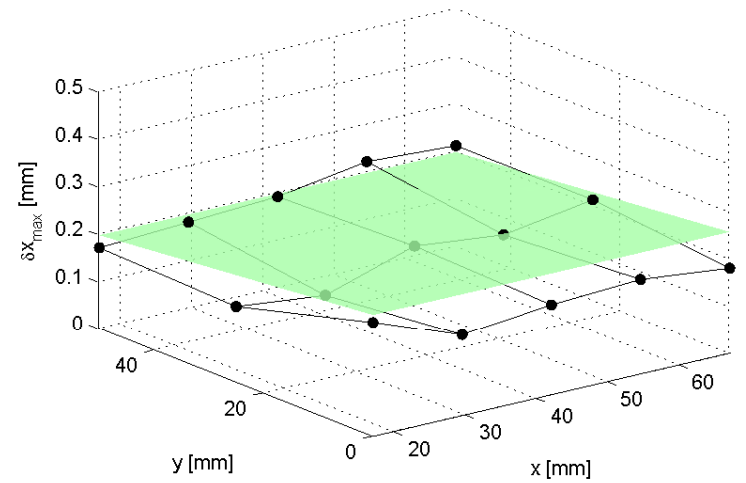

(a) Maximum error along $x$-axis

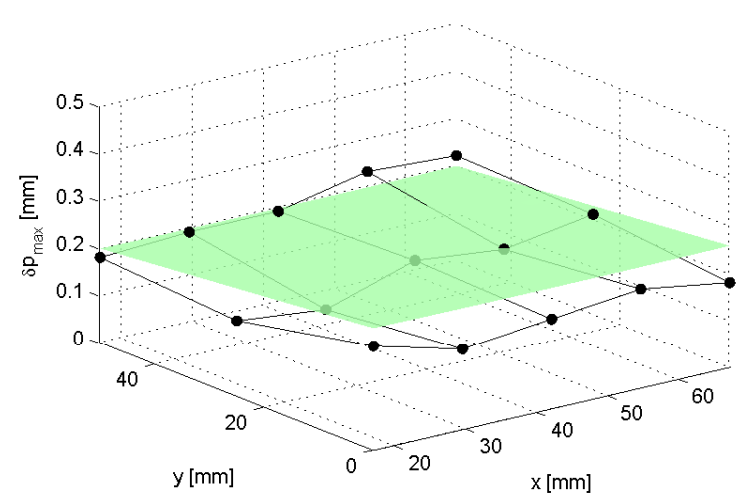

(c) Maximum positioning error

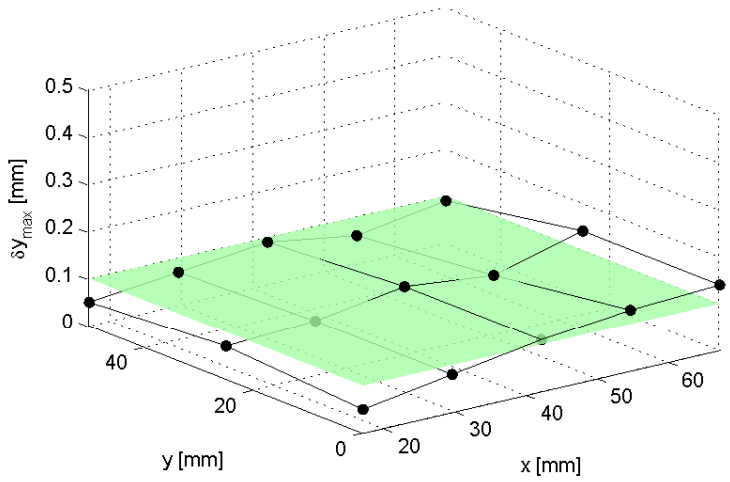

(b) Maximum error along $y$-axis

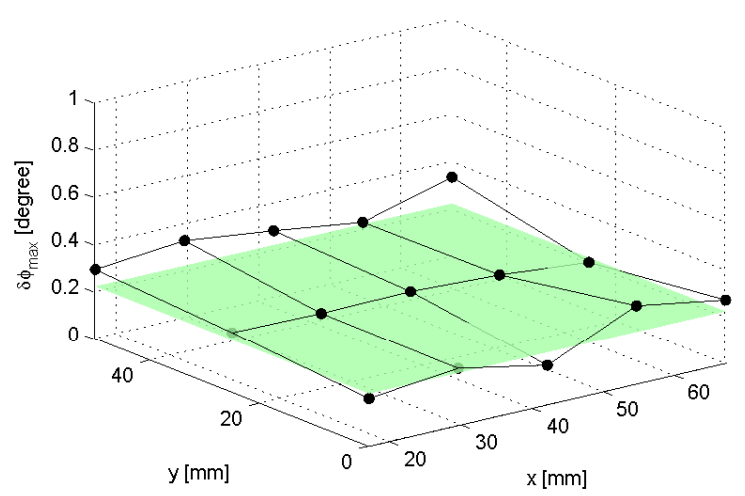

(d) Maximum orientation error

Fig. 9 Comparison of error distributions for Case 1 with a constant orientation $\phi=0$ : The green surface is obtained from simulation while black points from measurements.

due to the reason that angular measurement is more sensitive to the random error and influence of environments, etc, than the positional measurement.

The distributions of measured errors with constant orientation $\phi=\pi / 6$ are shown in Fig. 10. The simulated $\delta x_{\max }$, as shown in Fig. 10(a), varying from $0.224 \mathrm{~mm}$ to $0.253 \mathrm{~mm}$, has a distribution similar to that corresponding to $\phi=0$, while $\delta y_{\max }$ is constant along $y$-axis and increases gradually with the $x$-coordinate (see Fig. 10(b)), from $0.103 \mathrm{~mm}$ to $0.123 \mathrm{~mm}$. In Fig. 10(d), the simulated orientation error $\delta \phi_{\max }$ is constant and is equal to $0.264 \mathrm{deg}$. The positioning error $\delta p_{\max }$, varying from $0.226 \mathrm{~mm}$ to $0.268 \mathrm{~mm}$, increases when the measuring point moves from the upper left corner to the lower right corner throughout the workspace as displayed in Fig. 10(c). The RMSD values between the simulations and measurements of $\delta x, \delta y, \delta p$ and $\delta \phi$ are equal to $47 \mu \mathrm{m}, 34 \mu \mathrm{m}, 34 \mu \mathrm{m}$ and $0.103 \mathrm{deg}$, respectively. Therefore, the measurements have a good correlation with the simulations.

6.2 Error Distributions for Case 2. In Case 2, both prismatic and revolute joint clearances are considered. The distributions of the maximum pose errors are shown in Figs. 11 and 12. By comparing Fig. 11 to Fig. 9 , it can be found that the differences between the simulation results and measurements really depend on the orientation of the MP. For $\phi=0$, the RMSD values between the simulations and measurements are equal to $77 \mu \mathrm{m}, 62 \mu \mathrm{m}, 81 \mu \mathrm{m}$ and $0.086 \mathrm{deg}$, for $\delta x, \delta y, \delta p$ and $\delta \phi$, respectively. Although the difference between the simulations and experiments in Fig. 11(b) under the given scale and unit seems larger than the other results, however, the maximum value is around $0.5 \mathrm{deg}$ and the statistical analysis also shows that the difference is acceptable.

For $\phi=\pi / 6$, the RMSD values between the simulations and measurements of $\delta x, \delta y, \delta p$ and $\delta \phi$, are equal to $26 \mu \mathrm{m}, 36 \mu \mathrm{m}$, $34 \mu \mathrm{m}$ and $0.063 \mathrm{deg}$, respectively. Note that the correlation between the simulation results and the measurements is better with $\phi=\pi / 6$ than $\phi=0$.

6.3 Discussion on Measurement Results. As shown in Fig. 13, both for Cases 1 and 2, the measurement errors with $\phi=\pi / 6$ are larger than that of $\phi=0$, which agrees with the distributions obtained from simulations. Moreover, the sample standard deviations (SSD) of the measured orientation errors are equal to 0.052, 0.029, 0.086 and $0.034 \mathrm{deg}$, respectively. This means that the measured orientation errors have very small fluctuations among the discrete points. The positioning performance of the robot, namely, their accuracy, is defined in accordance to ISO 9283: 1998 [23] as:

$$
A P_{p}=\delta p, A P_{\phi}=\delta \phi
$$

where $\delta p$ and $\delta \phi$ are the measured pose errors defined in Sec. 5.2. Figure 14 shows the accuracy of the manipulator, where the measured points covering the maximum workspace are demonstrated in Fig. 12(a). The position accuracy in measured points is $0.2 \sim$ $0.35 \mathrm{~mm}$, while the orientation accuracy is $0.2 \sim 0.45 \mathrm{deg}$. 


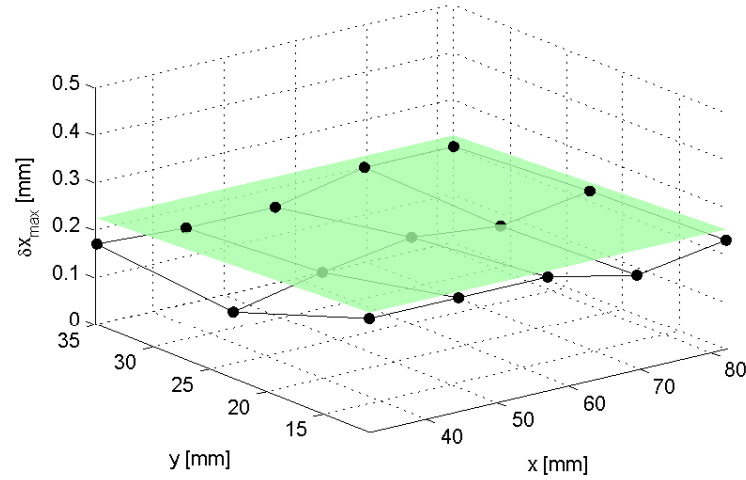

(a) Maximum error along $x$-axis

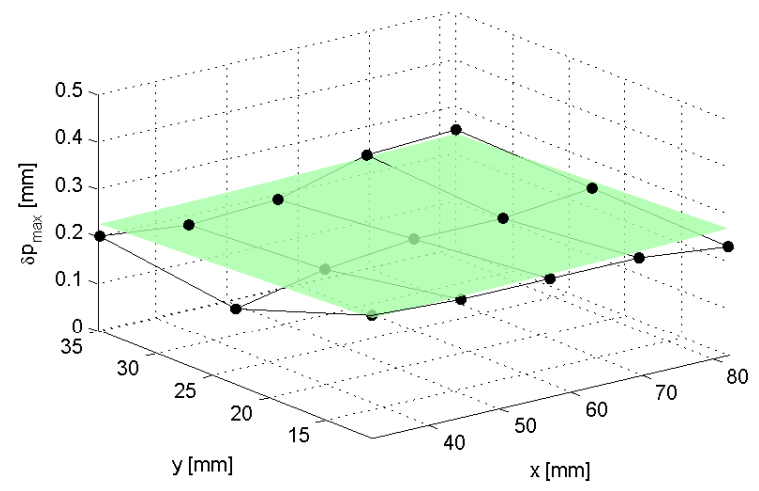

(c) Maximum positioning error

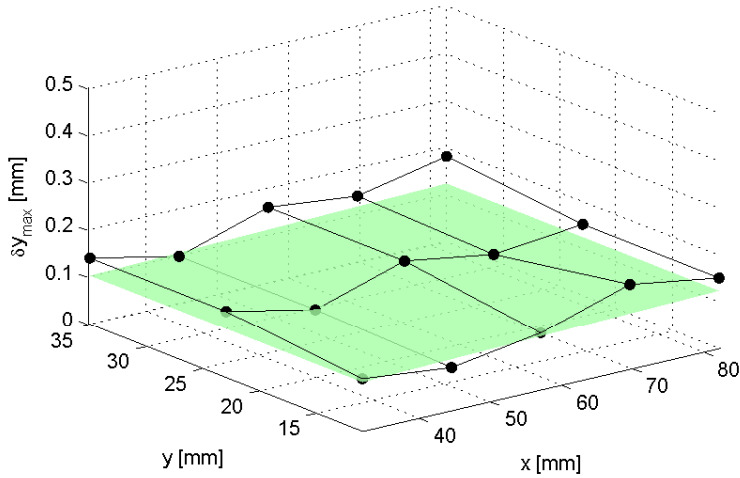

(b) Maximum error along $y$-axis

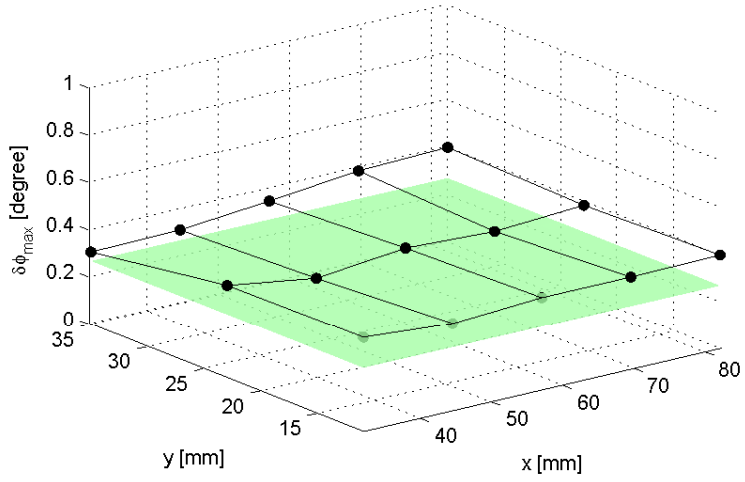

(d) Maximum orientation error

Fig. 10 Comparison of error distributions for Case 1 with a constant orientation $\phi=\pi / 6$.

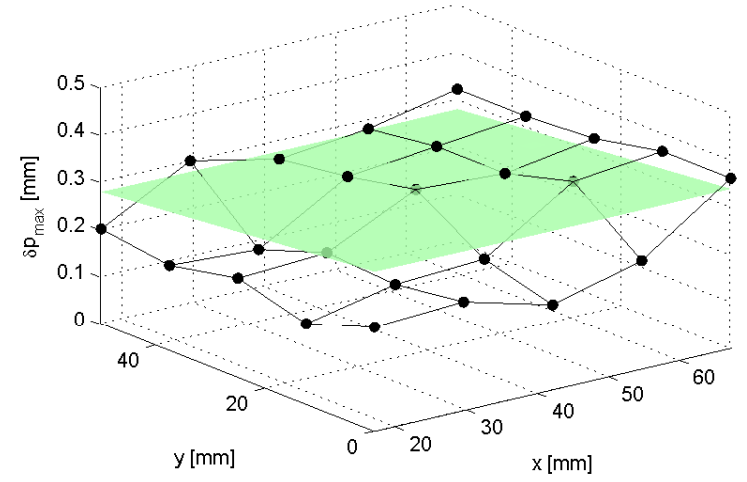

(a) Maximum positioning error

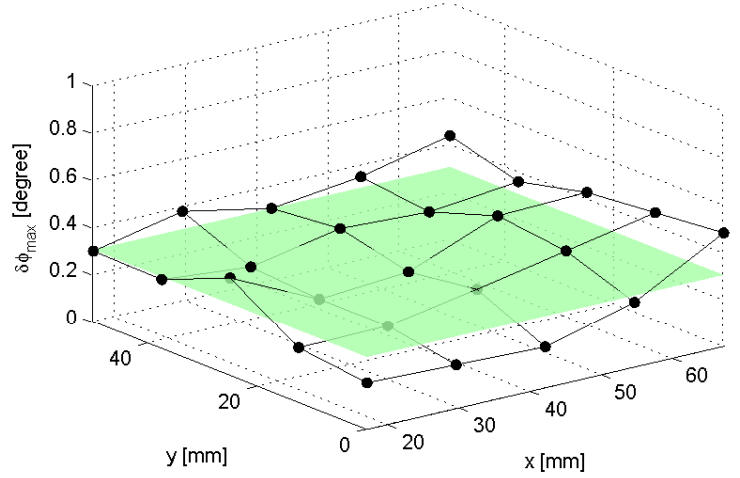

(b) Maximum orientation error

Fig. 11 Comparison of error distributions for Case 2 with a constant orientation $\phi=0$.

The experiments show that most of the measurements line along the boundaries established with the mathematical model, with a few exceptions. In order to evaluate the comparison between the simulations and measurements, we made a statistical regression analysis [24], as shown in Fig. 15 for Case 2 with a constant-orientation $\phi=\pi / 6$. Most of the simulation results are distributed in the measured error bands $\delta_{\text {mea }} \pm 2 A_{\text {mea }}$ except the orientation errors, where $\delta_{\text {mea }}$ is the measurement error defined in Sec. 5.2 and $A_{\text {mea }}$ is the accuracy of the measurement system in Sec. 5. The deviations in the simulation results are equal to $0.02 \mathrm{~mm}$ and $0.023 \mathrm{deg}$, respectively, as derived from the measurements of joint clearances. Although the simulated $\delta \phi$ are located beyond the measured error band for some points, the maximum difference between the two error bands is $0.086 \mathrm{deg}$, which means that the simulation results are quite close to the measurements. The possible reasons which cause disagreement between the simulations and measurements are random and systematic errors, the influence of the MP inclination and elastic deflection etc. 


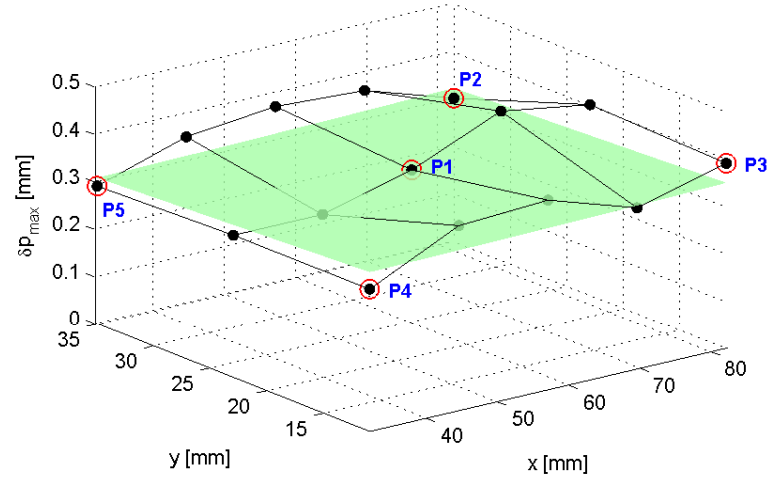

(a) Maximum positioning error

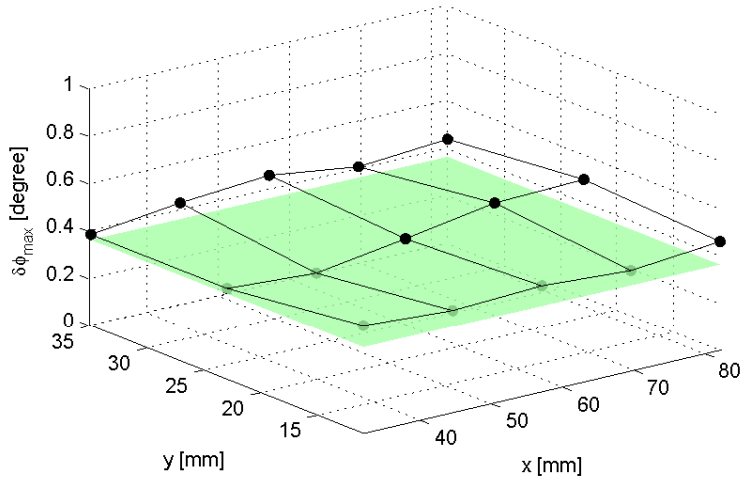

(b) Maximum orientation error

Fig. 12 Comparison of error distributions for Case 2 with a constant orientation $\phi=\pi / 6$.
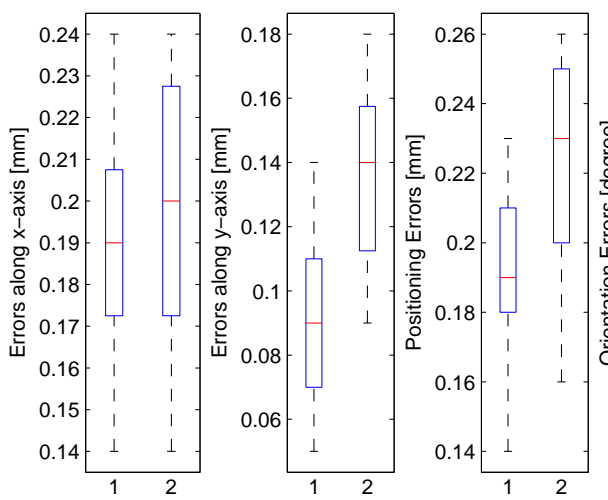

(a) Measurements of Case 1

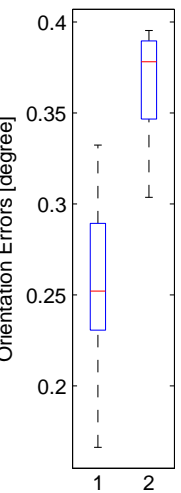

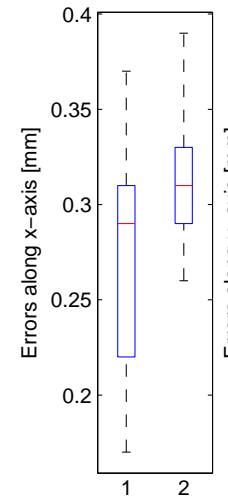
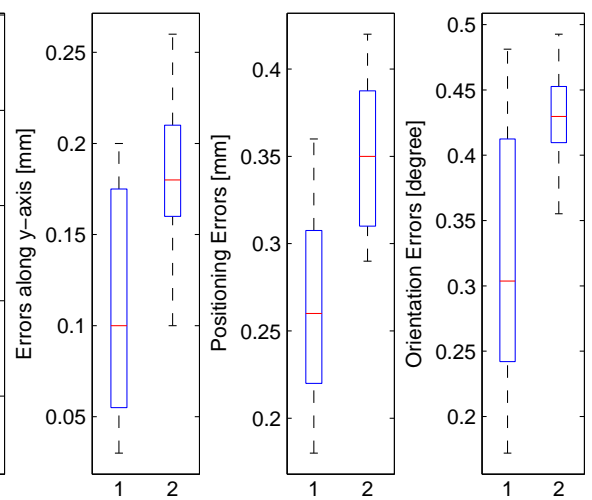

(b) Measurements of Case 2

Fig. 13 Boxplot of the measurements for Cases 1 and 2. No. 1 and 2 of horizontal axes stand for the measurements with constant-orientations $\phi=0$ and $\phi=\pi / 6$, respectively.
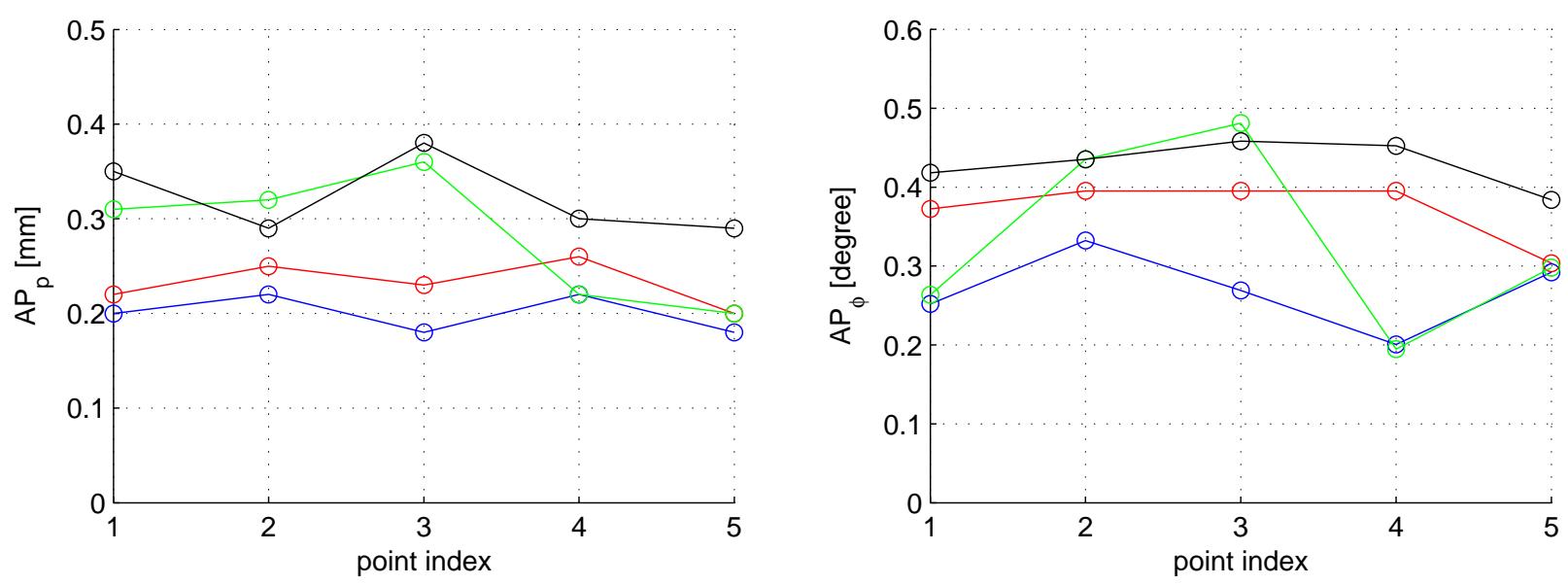

Fig. 14 Position and orientation accuracy at five poses.

\section{Conclusions}

In this paper, the pose error of a planar parallel manipulator was studied. A new error model was developed for PPMs with due considerations of both configuration errors and joint clearances. With the model, the pose error estimation problem was formulated as an optimization problem, which can estimate maximum pose errors. The error analysis method was deduced and explained in detail. This method can also be applied to planar serial mechanisms.

Another contribution lies in the experimental validation of the error model. Experiments were conducted to obtain the distribution of pose errors throughout the workspace, the results being compared with the errors estimated by the error model. It turns out that there is a good correlation between the pose error simulations and measurements. Moreover, the simulations show that the angular clearances in the passive prismatic joints have much more influence on the pose 

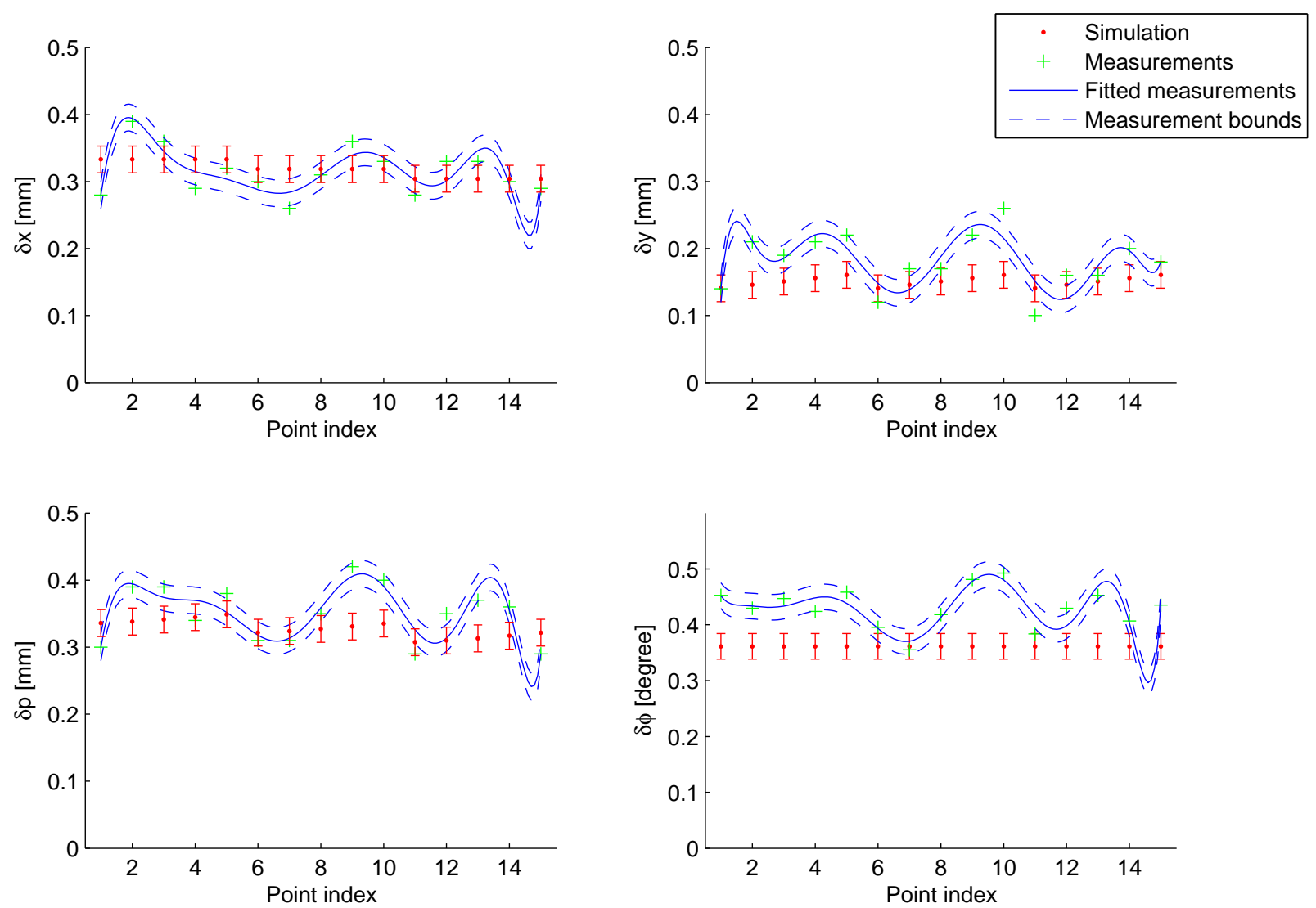

Fig. 15 Comparison between the measurements and simulation results for Case 2 with a constant orientation $\phi=\pi / 6$.

errors of the moving platform than the revolute joint clearances. This is associated with the experiments, in which the angular clearances in the linear bearings reach their tolerance bounds as much as possible. This suggests that one possible approach to eliminate the errors due to joint clearances is to preload the joint. The validated work can be used for error analysis and compensation in future work. Moreover, other error sources such as manufacturing errors will be considered.

\section{References}

[1] Ryu, J., and Cha, J., 2003. "Volumetric error analysis and architecture optimization for accuracy of HexaSlide type parallel manipulators". Mechanism and Machine Theory, 38, pp. 227-240.

[2] Liu, H., Huang, T., and Chetwynd, D. G., 2011. "A general approach for geometric error modeling of lower mobility parallel manipulators". ASME Journal of Mechanisms and Robotics, 3(2), pp. 021013-1-021013-13.

[3] Yu, A., Bonev, I. A., and Murray, P. Z., 2008. "Geometric approach to the accuracy analysis of a class of 3-DOF planar parallel robots". Mechanism and Machine Theory, 43, pp. 364-375.

[4] Briot, S., and Bonev, I. A., 2010. "Accuracy analysis of 3T1R fully-parallel robots". Mechanism and Machine Theory, 45, pp. 695-706.

[5] Lin, P. D., and Chen, J. F., 1992. "Accuracy analysis of planar linkages by the matrix method". Mechanism and Machine Theory, 35, pp. 507-516.

[6] Ting, K., Zhu, J., and Watkins, D., 2000. "The effects of joint clearance on position and orientation deviation of linkages and manipulators". Mechanism and Machine Theory, 35, pp. 391-401.

[7] Fogarasy, A., and Smith, M., 1998. "The influence of manufacturing tolerance on the kinematic performance of mechanisms". Proc. of Institution of Mechanical Engineers-Part C: Mechanical Engineering Science, 212, pp. 35-47.

[8] Lim, S. R., et al., 2002. "Error analysis of a parallel mechanism considering link stiffness and joint clearances". Journal of Mechanical Science and Technology, 16(6), pp. 799-809.

[9] Castelli, V. P., and Venanzi, S., 2005. "Clearance influence analysis on mechanisms". Mechanism and Machine Theory, 40, pp. 1316-1329.

[10] Venanzi, S., and Castelli, V. P., 2005. "A new technique for clearance influence analysis in spatial mechanisims". ASME Journal of Mechanical Design, 127, pp 446-455.

[11] Meng, J., Zhang, D., and Li, Z., 2009. "Accuracy analysis of parallel manipula- tors with joint clearance". ASME Journal of Mechanical Design, 131, pp. 0110131-011013-9.

[12] Binaud, N., Cardou, P., Caro, S., and Wenger, P.. "The Kinematic Sensitivity of Robotic Manipulators to Joint Clearance". Proc. of ASME Design Engineering Technical Conferences, August 15-18, 2010, Montreal, QC., Canada.

[13] Binaud, N., Caro, S., Bai, S., and Wenger, P., 2010. "Comparison of 3-PPR parallel planar manipulators based on their sensitivity to joint clearances". In Proc. IEEE/RSJ Inter. Conf. on Intelligent Robots and Systems (IROS), Taipei, October 18-22, pp. 2778-2783.

[14] Wei, W., and Simaan, N., 2010. "Design of planar parallel robots with preloaded flexures for guaranteed backlash prevention". ASME Journal of Mechanisms and Robotics, 2(1), pp. 011012-1-011012-10.

[15] Carusone, J., and D’Eleuterio, G. M., 1993. "Tracking control for end-effector position and orientation of structurelly flexible manipulators". Journal of Robotic Systems, 10(6), pp. 847-870.

[16] Zhuang, H., 1997. "Self-calibration of parallel mechanisms with a case study on Stewart platforms". IEEE Transactions on Robotics and Automation, 13(3), pp. 387-397.

[17] Voglewede, P., and Uphoff, I., 2004. "Application of workspace generation techniques to determine the unconstrained motion of parallel manipulators". ASME Journal of Mechanical Design, 126, pp. 283-290.

[18] Bai, S., and Caro, S., 2009. "Design and analysis of a 3-PPR planar robot with U-shape base". Proc. of 14th Inter. Conference on Advanced Robotics, Munich, Germany, June 22-26.

[19] SMAC Product Catalog version 2009 on the WWW, at http://www.smacmca.com/.

[20] Caro, S., Binaud, N., and Wenger, P., 2009. "Sensitivity analysis of 3-RPR planar parallel manipulators". Journal of Mechanical Design, 131, pp. 121005-1-13.

[21] HTTP://www.pdfsupply.com/pdfs/dvt-554c.pdf

[22] HTTP://www.cognex.com/support/files/DVT/Intellect_Manual.pdf

[23] International standard, Manipulating industrial robots-Performance criteria and related test methods, ISO 9283: 1998.

[24] Douglas C., M., 2009. "Design and analysis of experiments". 7th Edition, John Wiley \& Sons. 


\section{Appendix A}

The matrices in Eqn. (9) are given below

$$
\begin{aligned}
& \mathbf{H}_{a}=\operatorname{diag}\left[\mathbf{w}_{1}^{T} \mathbf{E}^{T} \mathbf{h}_{1} \mathbf{w}_{2}^{T} \mathbf{E}^{T} \mathbf{h}_{2} \mathbf{w}_{3}^{T} \mathbf{E}^{T} \mathbf{h}_{3}\right] \\
& \mathbf{H}_{\alpha}=\operatorname{diag}\left[\mathbf{w}_{1}^{T}\left(a_{1} \mathbf{h}_{1}+s_{1} \mathbf{u}_{1}+d_{1} \mathbf{v}_{1}\right)+l_{1} \mathbf{w}_{2}^{T}\left(a_{2} \mathbf{h}_{2}+s_{2} \mathbf{u}_{2}+d_{2} \mathbf{v}_{2}\right)+l_{2} \mathbf{w}_{3}^{T}\left(a_{3} \mathbf{h}_{3}+s_{3} \mathbf{u}_{3}+d_{3} \mathbf{v}_{3}\right)+l_{3}\right] \\
& \mathbf{H}_{\beta}=\operatorname{diag}\left[\mathbf{w}_{1}^{T}\left(s_{1} \mathbf{u}_{1}+d_{1} \mathbf{v}_{1}\right)+l_{1} \mathbf{w}_{2}^{T}\left(s_{2} \mathbf{u}_{2}+d_{2} \mathbf{v}_{2}\right)+l_{2} \mathbf{w}_{3}^{T}\left(s_{3} \mathbf{u}_{3}+d_{3} \mathbf{v}_{3}\right)+l_{3}\right] \\
& \mathbf{H}_{d}=\operatorname{diag}\left[\mathbf{w}_{1}^{T} \mathbf{E}^{T} \mathbf{v}_{1} \mathbf{w}_{2}^{T} \mathbf{E}^{T} \mathbf{v}_{2} \mathbf{w}_{3}^{T} \mathbf{E}^{T} \mathbf{v}_{3}\right] \\
& \mathbf{H}_{\gamma}=\operatorname{diag}\left[d_{1} \mathbf{w}_{1}^{T} \mathbf{v}_{1}+l_{1} d_{2} \mathbf{w}_{2}^{T} \mathbf{v}_{2}+l_{2} d_{3} \mathbf{w}_{3}^{T} \mathbf{v}_{3}+l_{3}\right] \\
& \mathbf{H}_{\theta}=\operatorname{diag}\left[l_{1} l_{2} l_{3}\right] \\
& \mathbf{H}_{\rho}=\operatorname{diag}\left[\mathbf{w}_{1}^{T} \mathbf{E}^{T} \mathbf{n}_{1} \mathbf{w}_{2}^{T} \mathbf{E}^{T} \mathbf{n}_{2} \mathbf{w}_{3}^{T} \mathbf{E}^{T} \mathbf{n}_{3}\right] \\
& \mathbf{H}_{r}=\operatorname{diag}\left[\mathbf{w}_{1}^{T} \mathbf{E}^{T} \mathbf{k}_{1} \mathbf{w}_{2}^{T} \mathbf{E}^{T} \mathbf{k}_{2} \mathbf{w}_{3}^{T} \mathbf{E}^{T} \mathbf{k}_{3}\right] \\
& \mathbf{H}_{\psi}=\operatorname{diag}\left[r_{1} \mathbf{w}_{1}^{T} \mathbf{k}_{1} r_{2} \mathbf{w}_{2}^{T} \mathbf{k}_{2} r_{3} \mathbf{w}_{3}^{T} \mathbf{k}_{3}\right]
\end{aligned}
$$

The matrices $\mathbf{J}_{A}, \mathbf{J}_{B}, \mathbf{J}_{C}$, and $\mathbf{J}_{D}$ in Eqn. (13) are expressed as

$$
\begin{aligned}
& \mathbf{J}_{A}=\left[\begin{array}{ll}
\mathbf{J}_{a} & \mathbf{J}_{\alpha}
\end{array}\right]\left[\begin{array}{ll}
\mathbf{A}_{a} & \mathbf{A}_{\alpha}
\end{array}\right]_{6 \times 6}^{-1}, \mathbf{A}_{a}=\operatorname{diag}\left[\mathbf{h}_{1} \mathbf{h}_{2} \mathbf{h}_{3}\right], \mathbf{A}_{\alpha}=\operatorname{diag}\left[\begin{array}{lll}
a_{1} \mathbf{E h}_{1} & a_{2} \mathbf{E h}_{2} & a_{3} \mathbf{E h}_{3}
\end{array}\right] \\
& \mathbf{J}_{B}=\left[\begin{array}{ll}
\mathbf{0}_{3 \times 3} & \mathbf{J}_{\beta}
\end{array}\right]\left[\begin{array}{ll}
\mathbf{B}_{s} & \mathbf{B}_{\beta}
\end{array}\right]_{6 \times 6}^{-1}, \mathbf{B}_{s}=\operatorname{diag}\left[\begin{array}{lll}
\mathbf{u}_{1}^{\prime} & \mathbf{u}_{2}^{\prime} & \mathbf{u}_{3}^{\prime}
\end{array}\right], \mathbf{B}_{\beta}=\operatorname{diag}\left[\begin{array}{llll}
s_{1} & \mathbf{E} \mathbf{u}_{1}^{\prime} & s_{2} \mathbf{E} \mathbf{u}_{2}^{\prime} & s_{3} \\
\mathbf{E u} & \mathbf{u}_{3}^{\prime}
\end{array}\right]
\end{aligned}
$$

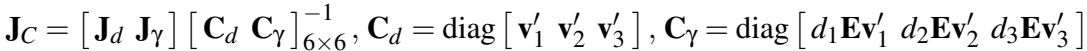

$$
\begin{aligned}
& \mathbf{J}_{D}=\left[\begin{array}{ll}
\mathbf{J}_{r} & \mathbf{J}_{\psi}
\end{array}\right]\left[\begin{array}{ll}
\mathbf{D}_{r} & \mathbf{D}_{\psi}
\end{array}\right]_{6 \times 6}^{-1}, \mathbf{D}_{r}=\operatorname{diag}\left[\mathbf{k}_{1}^{\prime} \mathbf{k}_{2}^{\prime} \mathbf{k}_{3}^{\prime}\right], \mathbf{D}_{\psi}=\operatorname{diag}\left[\begin{array}{llll}
r_{1} & \mathbf{E} \mathbf{k}_{1}^{\prime} & r_{2} \mathbf{E} \mathbf{k}_{2}^{\prime} & r_{3} \mathbf{E k}_{3}^{\prime}
\end{array}\right]
\end{aligned}
$$

with

$$
\mathbf{u}_{i}^{\prime}=\left[\begin{array}{c}
\cos \beta_{i} \\
\sin \beta_{i}
\end{array}\right], \mathbf{v}_{i}^{\prime}=\left[\begin{array}{c}
\cos \gamma_{i} \\
\sin \gamma_{i}
\end{array}\right], \mathbf{k}_{i}^{\prime}=\left[\begin{array}{c}
\cos \psi_{i} \\
\sin \psi_{i}
\end{array}\right]
$$

